\title{
A case study of ACV variables discovered in the Zwicky Transient Facility survey ${ }^{\star}$
}

\author{
N. Faltová ${ }^{1}$, K. Kallová ${ }^{1}$, M. Prišegen ${ }^{1}$, P. Staněk ${ }^{1}$,J. Supíková ${ }^{2}$, C. Xia ${ }^{1}$, K. Bernhard ${ }^{3,4}$, \\ S. Hümmerich ${ }^{3,4}$, and E. Paunzen ${ }^{1}$ \\ ${ }^{1}$ Department of Theoretical Physics and Astrophysics, Faculty of Science, Masaryk University, Kotlářská 2, 61137 Brno, \\ Czech Republic \\ e-mail: epaunzen@physics.muni.cz \\ 2 Faculty of Informatics, Masaryk University, Brno, Czech Republic \\ 3 Bundesdeutsche Arbeitsgemeinschaft für Veränderliche Sterne e.V. (BAV), Berlin, Germany \\ ${ }^{4}$ American Association of Variable Star Observers (AAVSO), Cambridge, USA
}

Received 12 June 2021 / Accepted 17 August 2021

\begin{abstract}
Context. Magnetic chemically peculiar (mCP) stars exhibit complex atmospheres that allow the investigation of the interplay of atomic diffusion, magnetic fields, and stellar rotation. A non-uniform surface distribution of chemical elements and the non-alignment of the rotational and magnetic axes result in the variability of several observables. Photometrically variable mCP stars are referred to as $\alpha^{2}$ Canum Venaticorum (ACV) variables.

Aims. The present work presents a case study of known variables from the Zwicky Transient Facility (ZTF) survey, with the aim of investigating the survey's suitability for the detection and study of new ACV variables.

Methods. Using suitable selection criteria based on the known characteristics of ACV variables, candidate ACV stars were selected from the ZTF Catalog of Periodic Variable Stars. All light curves were inspected in detail to select the most promising candidates. Where available, low-resolution spectra from the Large Sky Area Multi-Object Fiber Spectroscopic Telescope (LAMOST) were employed to classify the stars on the MK system and confirm their status as mCP stars.

Results. We have identified 86 new promising ACV star candidates. Fifteen of these stars have LAMOST spectra available that, in all cases, confirm them as classical mCP stars, which highlights the viability of our approach. We present astrophysical parameters for all sample stars that can be sorted into four subgroups characterized by distinct light curve shapes. Anti-phase variations in different photometric passbands, in particular, are a unique characteristic of a subset of ACV stars readily usable for their identification. The availability of data in three different passbands $(g, r$, and $i$ ) is a major advantage of the ZTF survey.

Conclusions. On the basis of our experience with other photometric surveys and the analysis of light curves, we conclude that the ZTF is well suited to the search for, and the analysis of, ACV variables that are not considered in the available ZTF variable star catalogs. Further work will focus on the development and refinement of a search algorithm to correctly identify these stars in ZTF data and, subsequently, in massive photometric time series databases in general.
\end{abstract}

Key words. stars: chemically peculiar - stars: variables: general - stars: rotation - binaries: eclipsing

\section{Introduction}

The magnetic chemically peculiar (mCP) stars, which encompass the groups of the Ap/CP2 stars and the He-peculiar stars (Preston 1974), are upper main-sequence objects (spectral types B to early F) that exhibit anomalous surface abundances and a non-uniform distribution of chemical elements. They are further characterized by the presence of a strong and global magnetic field. The CP2 stars show overabundances of $\mathrm{Si}, \mathrm{Sr}, \mathrm{Cr}$, $\mathrm{Eu}$, and the rare-earth elements as compared to the solar composition (Preston 1974; Saffe et al. 2005). The He-peculiar stars comprise the B5 to B9 He-weak (CP4) stars that show anomalously weak $\mathrm{He}$ I lines for their temperature type and the more massive B1 to B3 He-strong stars with anomalously strong He I lines (Andrillat \& Jaschek 1998).

In addition, there are also the CP1 stars (the metallic-line or Am/Fm stars; Qin et al. 2019) and the CP3 stars (the mercury-

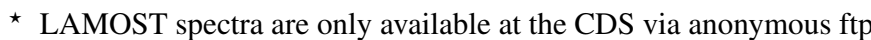
to cdsarc.u-strasbg. fr (130.79.128.5) or via http://cdsarc. u-strasbg.fr/viz-bin/cat/J/A+A/656/A125 manganese or HgMn stars; Paunzen et al. 2021). These groups are believed to possess only very weak, if any, magnetic fields (Bychkov et al. 2003).

The non-uniform distribution of chemical elements characteristic for $\mathrm{mCP}$ stars is responsible for the formation of spots and patches of enhanced element abundances on their surfaces (Michaud et al. 1981). Several mechanisms and their interplay may be responsible for the development of these spots, such as a weak stellar wind (Krtička 2016), the characteristics of the stellar magnetic field (Romanyuk 2014), the occurrence of a weak convective zone, and the implications of slow rotation.

As a result of the abundance inhomogeneities, the rotation of mCP stars necessarily results in periodic variations of the observed flux (known as photometric spots; Krtička et al. 2007) and periodic changes in their spectra. This variability can be explained by the oblique rotator model, which was proposed by Babcock (1949) and developed by Stibbs (1950). The period of the observed variations is hence identical with the rotational period of an mCP star. $\alpha^{2}$ Canum Venaticorum was the first CP2 star that was identified as a photometric variable (Guthnik \& Prager 1914), which is the reason why photometrically variable $\mathrm{mCP}$ stars are 
generally referred to as $\alpha^{2}$ Canum Venaticorum (ACV) variable stars (Samus' et al. 2017).

Several elements play a part in the observed variations. For instance, Krtička et al. (2007) demonstrated that the variability of the He-strong star HD 37776 is caused by spots of $\mathrm{He}$ and $\mathrm{Si}$, whereas Krtička et al. (2009) produced evidence that the variability of the CP2 star HR 7224 is the result of an inhomogeneous surface distribution of $\mathrm{Fe}$ and $\mathrm{Si}$. Other elements such as $\mathrm{Si}, \mathrm{Cr}$, Sr, or Eu may contribute to the observed variations in CP2 stars (Bernhard et al. 2015a).

The ACV variable stars are perfectly suited for direct measurement of the rotational period without the need for any additional calibrations. With astrophysical parameters such as stellar masses, effective temperature, radii, inclinations, and critical rotational velocities, it is possible to analyze the conservation of angular momentum during the main-sequence evolution (Netopil et al. 2017).

Although many excellent data sources on stellar variability are now available, the class of the ACV variables is only rarely considered in the algorithms employed for the automatic classification of variable stars into astrophysically meaningful classes (Blomme et al. 2010; Kügler et al. 2015; Bass \& Borne 2016). In this paper we present a case study of new ACV variables discovered in data from the Zwicky Transient Facility (ZTF), and show that the accuracy and cadence of the ZTF allows us to detect previously unknown stars of this kind. This survey reaches fainter magnitudes than most corresponding programs (Hümmerich et al. 2018), which means that we can probe the population of ACV variables at larger distances from the Sun. To date, the automatic detection and classification methods employed with the ZTF data have not included this class of variable stars (Chen et al. 2020; Ofek et al. 2020).

\section{Data selection and time series analysis}

The ZTF is a time-domain survey that is located at Palomar Observatory and in operation since 2017. It is a successor of the successful Palomar Transient Factory (PTF). The ZTF camera employs e2v CCD231-C6 devices and is mounted on the Palomar 48-inch Samuel Oschin Schmidt Telescope. It is able to scan 3750 square degrees an hour in three different passbands $(g, r$, and $i$ ) to a limiting magnitude of $20.5 \mathrm{mag}$. The main aim is to discover young supernovae and other types of transients. With its current strategy, the ZTF survey provides nearly 300 observations each year per object in the northern hemisphere. ZTF data are therefore ideally suited for studies of variable stars, binaries, AGN, and asteroids (Bellm et al. 2019; Masci et al. 2019).

Chen et al. (2020) exploited the ZTF Data Release 2 and compiled a catalog of 781602 periodic variable stars (The Zwicky Transient Facility Catalog of Periodic Variable Stars ${ }^{1}$ ). We systematically searched this catalog for candidate ACV variables by examining the light curves of an extended sample of stars in detail. Since the catalog does not contain a category for ACV-type variables, it can be assumed that any such objects were assigned to other categories of short-period variables, especially to the class of the RS Canum Venaticorum (RS CVn) stars which was the focus of our analysis. RS $\mathrm{CVn}$ stars are rotational variables whose light curves, at least on first impression, look similar to the light curves of ACV variables (Kozhevnikova \& Alekseev 2015). They are, however, close binary stars with active chromospheres and enhanced spot activity (Eaton et al. 1993).

\footnotetext{
1 http://variables.cn: 88
}

As there are 81393 RS CVn stars listed in Chen et al. (2020), further restrictions were made based on the known characteristics of the group of ACV variables (Netopil et al. 2017; Jagelka et al. 2019): (i) photometric period between one and ten days; (ii) amplitude in the $r$ band smaller than $0.3 \mathrm{mag}$; (iii) presence of a single independent variability frequency and corresponding harmonics; (iv) stable or marginally changing light curve throughout the covered time span; (v) $T_{\text {eff }}$ (taken from Andrae et al. 2018) between $6000 \mathrm{~K}$ and $25000 \mathrm{~K}$.

Items (iii) and (iv) are helpful to distinguish ACV variables from, respectively, pulsators (which are often multi-periodic) and other types of rotational variables, such as RS CVn stars. In contrast to ACV variables, whose light curves remain stable over very long periods of time (decades or more), most spotted stars show significant light changes as spots form and decay.

A total of 1400 objects passed these selection criteria, whose light curves were downloaded from the ZTF website ${ }^{2}$ and visually inspected. The most promising ACV candidates were selected, which resulted in the 86 candidates presented in this paper. The big advantage of the ZTF survey is that photometric data are available in at least two filters, which facilitates the differentiation from other similar groups of variable stars, such as the ellipsoidal variables.

The published periods by Chen et al. (2020) were inspected using the program package PERIOD04 (Lenz \& Breger 2005), which performs a discrete Fourier transform. Furthermore, we used the CLEANEST and phase dispersion minimization (PDM) algorithms as implemented in the program package PERANSO (Paunzen \& Vanmunster 2016). The same results were obtained within the derived errors, which depend on the time series characteristics (i.e. the distribution of the measurements over time and photon noise).

The complete set of phased light curves in the available filters is presented in Table A.1.

\section{Sample characteristics}

In the upper panel of Fig. 1, the distribution of the stars on the sky is shown. All stars are located in the Galactic disk with distances between 1000 and $6000 \mathrm{pc}$ from the Sun. They are also, on average, fainter than the known ACV variables (see Fig. 1, lower panel) investigated with other ground-based surveys (Bernhard et al. 2015a,b, 2020).

\subsection{Spectral classification}

In order to validate the selection process of our target star sample (Sect. 2) and assess its efficiency, we searched for matches in DR7 of the Large Sky Area Multi-Object Fiber Spectroscopic Telescope (LAMOST) database (Zhao et al. 2012).

The LAMOST project employs a four-meter quasi-meridian reflecting Schmidt telescope capable of observing up to 4000 targets per exposure simultaneously in a field of view of $5^{\circ}$ (Cui et al. 2012). Recently, in March 2020, the DR7 released more than ten million low-resolution spectra in the wavelength range 3690-9100 $\AA$, with a resolution of $R \sim 1800$.

In total, we found spectra for 15 stars, of which five (ZTF $\mathrm{J} 040517.81+335007.7, \quad \mathrm{~J} 050755.19+495727.2, \quad \mathrm{~J} 054009.21+$ 120945.1, J183551.46+334749.7, and $\mathrm{J} 212905.49+142755.5$ ) have more than one spectrum available. Only the spectrum with the highest signal-to-noise ratio was considered for the analysis in these cases. We note that the available sample is not biased by

\footnotetext{
2 https://www.ztf.caltech.edu/
} 

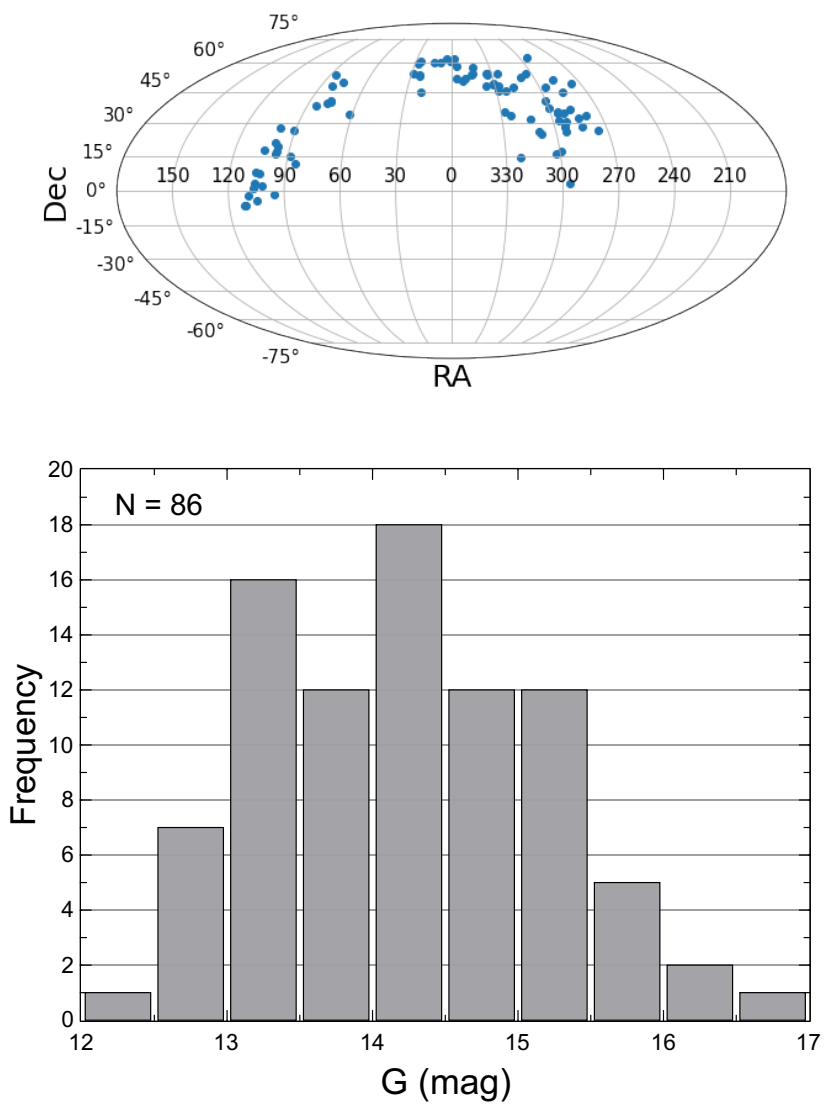

Fig. 1. Mollweide projection in right ascension and declination (upper panel) and the $G$ magnitude distribution (lower panel) of our sample stars. All stars are located within the Galactic disk.

the spectral type range, but by the apparent magnitude. It should therefore be a representative sample of 86 ACV stars.

The spectral classification was performed in the framework of a revised MK system by visually comparing, with the help of a simple graphical program, spectra in the spectral range from $3700 \AA$ to $9100 \AA$ to the spectra of standard stars using the notation of Gray \& Garrison (1987, 1989a,b), and Garrison \& Gray (1994). As ACV stars are mainly early B- to early F-type mainsequence stars, the spectral range of the standard star spectra was chosen to extend from $3800 \AA$ to $4600 \AA$ because the majority of the relevant spectral lines are situated in this wavelength region.

In the spectral classification we focused on several relevant indicators: the profile of the $\mathrm{H}$ lines, the ratio of the $\mathrm{Ca}$ II $\mathrm{K}$ to the $\mathrm{H} \varepsilon$ line, and the presence and strength of several well-known metallic lines (Hümmerich et al. 2020). The spectra of all of our sample stars show the characteristic signature of mCP stars: the well-known flux depression at around $5200 \AA$ (Kupka et al. 2003, 2004). The $\mathrm{Ca}$ II $\mathrm{K} / \mathrm{H} \varepsilon$ line ratio lies in the range $0.3-0.75$ for all of our sample stars, which is typical for the spectral classes A0 to A5. In most cases the $\mathrm{H}$ lines present the broad profiles of luminosity class $\mathrm{V}$ objects, confirming that the majority of our sample stars belong to the main sequence. The two hotter stars in our sample (J050755.19+495727.1 and J053725.00+382012.2) show narrow H-line profiles best fit by standards of luminosity class III.

The spectral classes in our sample are listed in Table 1. Peculiarities are listed in order of importance. Peculiarity types in parentheses or followed by a colon denote, respectively, mild or questionable peculiarities. With the two exceptions mentioned
Table 1. Spectral types of the 15 sample stars with spectra in LAMOST DR7.

\begin{tabular}{lccl}
\hline \hline LAMOST_ID & Obs_ID & $S / N$ & Spectral type \\
\hline J012145.51+443625.8 & 168003189 & 98 & kA1 hA5 V SrEu(Cr) \\
J040517.81+335007.7 & 189415040 & 37 & kA0 hA5 V EuSrCr \\
J050755.19+495727.1 & 290903161 & 64 & A0 III SiSrCrEu \\
J051331.05+393032.4 & 294302231 & 103 & kA1 hA7 V SrEuCr \\
J052856.69+475711.7 & 253703006 & 67 & kA1 hA7 V SrCrEu \\
J053725.00+382012.2 & 316409236 & 87 & A0 III SiEuSrCr \\
J054009.21+120945.0 & 476111206 & 106 & kA2 hA3 V SrCrEu \\
J055527.76+152315.3 & 98805041 & 88 & kA2 hA5 V SrCrEu \\
J065551.61+174248.1 & 372816239 & 75 & kA1 hA7 V SrCrEu \\
J182441.87+485404.6 & 155808159 & 17 & kA0: hA5: V SrCrEu \\
J190408.38+445439.1 & 362115243 & 110 & kA0 hA6 V EuCrSr(Si:) \\
J190831.72+511959.7 & 575716189 & 153 & kA0 hA3 V EuSrCr(Si:) \\
J194356.39+470831.0 & 572806020 & 96 & kA1 hA5 V SrEu(Cr) \\
J212905.49+142755.4 & 592409127 & 113 & kA1 hA7 V SrCrEu(Si:) \\
J213347.82+451647.6 & 476310217 & 51 & kA2 hA7 V SrCrEu \\
\hline
\end{tabular}

Notes. Also indicated are the LAMOST observation ID (Obs_ID) and the signal-to-noise ratio in the $g$ band $(\mathrm{S} / \mathrm{N})$.

above, all objects are cool mCP stars, which is also reflected by their astrophysical parameters (Sect. 3.2). Example spectra are provided in Fig. 2.

Cool CP2 stars and CP1 stars overlap in the temperature domain, and care needs to be taken in the differentiation of their spectra, which may look similar at first glance. CP1 stars are characterized by significant underabundances of $\mathrm{Ca}$ and $\mathrm{Sc}$ and general overabundances of iron-peak and heavier elements (Gray \& Corbally 2009). Therefore, in relation to the spectral type as derived from the hydrogen lines, they show weak Ca II $\mathrm{K}$ lines and a pronounced metallic-line spectrum, which also applies to most cool CP2 stars.

The enhancement of heavy elements in CP1 stars is general, whereas selected elements generally dominate the spectra of CP2 stars. Strong lines of the iron-peak element Cr are a common feature of both groups; strongly enhanced lines (e.g., Si and $\mathrm{Eu}$ ), however, are a diagnostic feature of CP2 stars, which also generally show broader and more diffuse Ca II K lines. Figure 3 illustrates the subtle differences between the spectra of a CP1 star (Qin et al. 2019) and a CP2 star of the present sample in the traditional blue-violet spectral region. While Sr II $4077 \AA$ is strong in both stars, the Sr II $4216 \AA$ line, which is used to confirm Sr peculiarity (Gray \& Corbally 2009), is only enhanced in the CP2 star.

More important, however, is the morphology of the $5200 \AA$ region. CP2 stars show a characteristic flux depression around $5200 \AA$, which is not observed in CP1 stars (Paunzen et al. 2005), and is therefore an important criterion to differentiate between these groups of objects.

Some of the stars, for example LAMOST J190408.37+445439.3, are very peculiar objects whose general spectral pattern resembles that of HD 101065 (Przybylski's Star), which is a rapidly oscillating Ap (roAp) star with a unique abundance pattern showing, for example, lines of Pm I, Pm II, Tc I, and Tc II (Hubrig et al. 2018). The cadence and accuracy of the ZTF measurements does not allow us to search for roAp pulsations, which have periods below $30 \mathrm{~min}$ and amplitudes of only a few mmags (Cunha et al. 2019). Unfortunately, most of our sample stars are also too faint to be observed by the TESS satellite (Sikora et al. 2019). Therefore, new ground-based observations are needed to search for this 


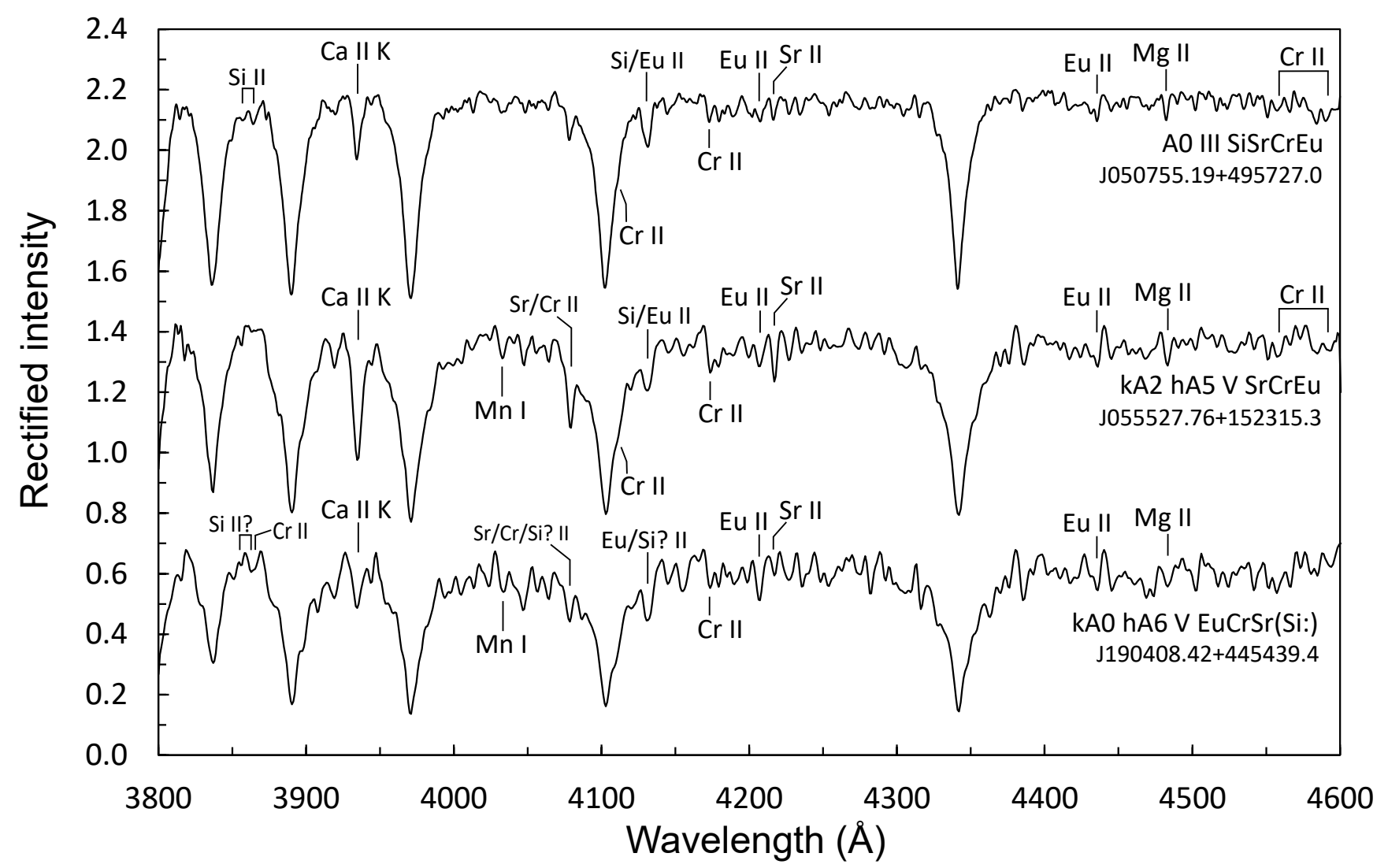

Fig. 2. Blue-violet spectral region of three newly identified mCP stars, based on spectra from LAMOST DR7. Some prominent lines of interest are identified.

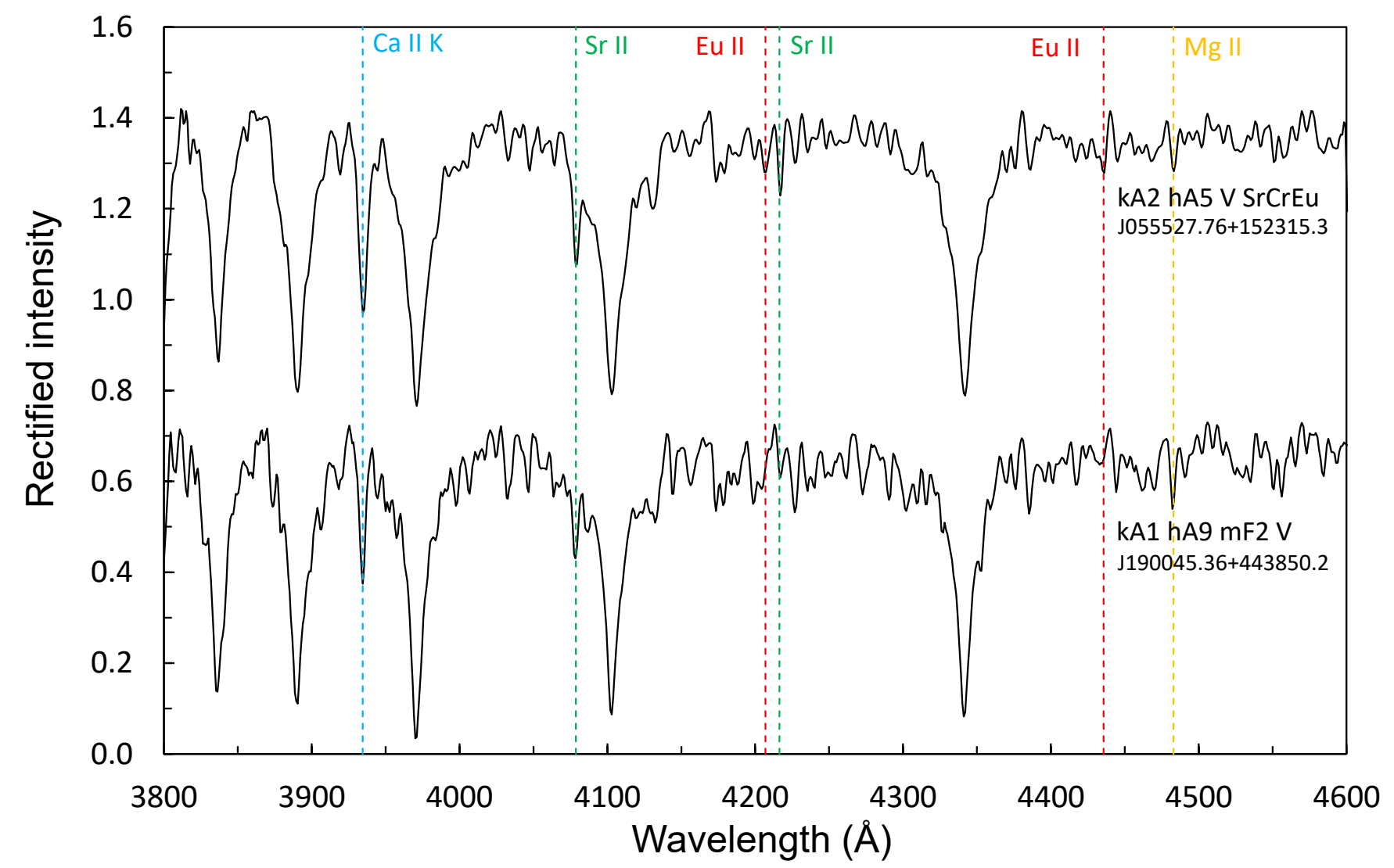

Fig. 3. Spectra of a CP2 star (upper panel) of the present sample and a CP1 star (lower panel) taken from Qin et al. (2019) in the traditional blue-violet spectral region. Some prominent lines of interest are identified. 
type of variability in our sample stars (Paunzen et al. 2015a, 2018).

\subsection{Astrophysical parameters}

As the next step, we determined and analyzed the astrophysical parameters of our sample stars, namely the effective temperature and luminosity, from which we deduced the evolutionary status.

As our sample stars are members of the Galactic disk and are situated beyond $1000 \mathrm{pc}$ from the Sun, interstellar reddening (absorption) needs to be taken into account. Unfortunately, Strömgren-Crawford indices, which allow a reliable reddening estimation, are not available for our sample stars (Paunzen 2015). We therefore relied on the reddening map of Green et al. (2019) and on the Gaia EDR3 photogeometric distances and their errors from Bailer-Jones et al. (2021) in order to interpolate within this map.

To derive the $T_{\text {eff }}$ values, we used the photometric data from 2MASS (Skrutskie et al. 2006), APASS (Henden \& Munari 2014), and Gaia DR2 (Arenou et al. 2018). Then several independent calibrations were applied, as listed in Paunzen et al. (2006, 2017), and Netopil et al. (2008). Furthermore, all available photometric data (ultraviolet, optical, and near-infrared bands) were employed to analyze the spectral energy distribution (SED) of our sample stars. For the SED fitting, the VO SED Analyzer was used (Bayo et al. 2008, VOSA), which is a browser-based online tool. The photometric data were derived from the available VO catalogs. Two different stellar atmosphere models were used to determine the effective temperatures: the blackbody model and the Kurucz ATLAS9 model (Castelli et al. 1997) using new opacity distribution functions (Castelli \& Kurucz 2003) and solar metallicity. The derived values are generally in good agreement with the photometrically calibrated values. The errors were derived by calculating the standard deviations from all available individual $T_{\text {eff }}$ values.

The luminosity of our target stars was calculated using the photogeometric distances and their errors (directly transformed to the error for the luminosity) from Bailer-Jones et al. (2021) and the bolometric corrections from Flower (1996). ZTFJ232952.58+501457.3 was excluded from further analysis because of its large luminosity uncertainty.

Ages, masses, and evolutionary phases were estimated by the Stellar Isochrone Fitting Tool ${ }^{3}$, which follows the methods described by Malkov et al. (2010). PARSEC isochrones (Bressan et al. 2012) for a solar metallicity of $[Z]=0.020$ were used in this context. We favor this value because it has been shown to be consistent with most recent results of helioseismology (Buldgen 2019; Vagnozzi 2019).

In Fig. 4 the distributions of the $T_{\text {eff }}$, mass, and evolutionary phase values are shown. The last are taken from the evolutionary models by Bressan et al. (2012), and Chen et al. (2014). They divided their tracks and isochrones in 15 phases from the premain sequence to the thermally pulsing AGB stage, respectively. In line with the spectral classifications presented in Sect. 3.1, most of the stars have $T_{\text {eff }}$ values between 7300 and $8500 \mathrm{~K}$ (spectral types between F0 and A3). The masses are also consistent with the derived spectral types. Except for a single star (ZTFJ071633.43-020221.4), all objects are located between the zero age main sequence (ZAMS, phase 5) and the terminal age main sequence (TAMS, phase 6). Therefore, they are all hydrogen-burning main-sequence objects, which is additional

\footnotetext{
3 https://github.com/Johaney-s/StIFT
}
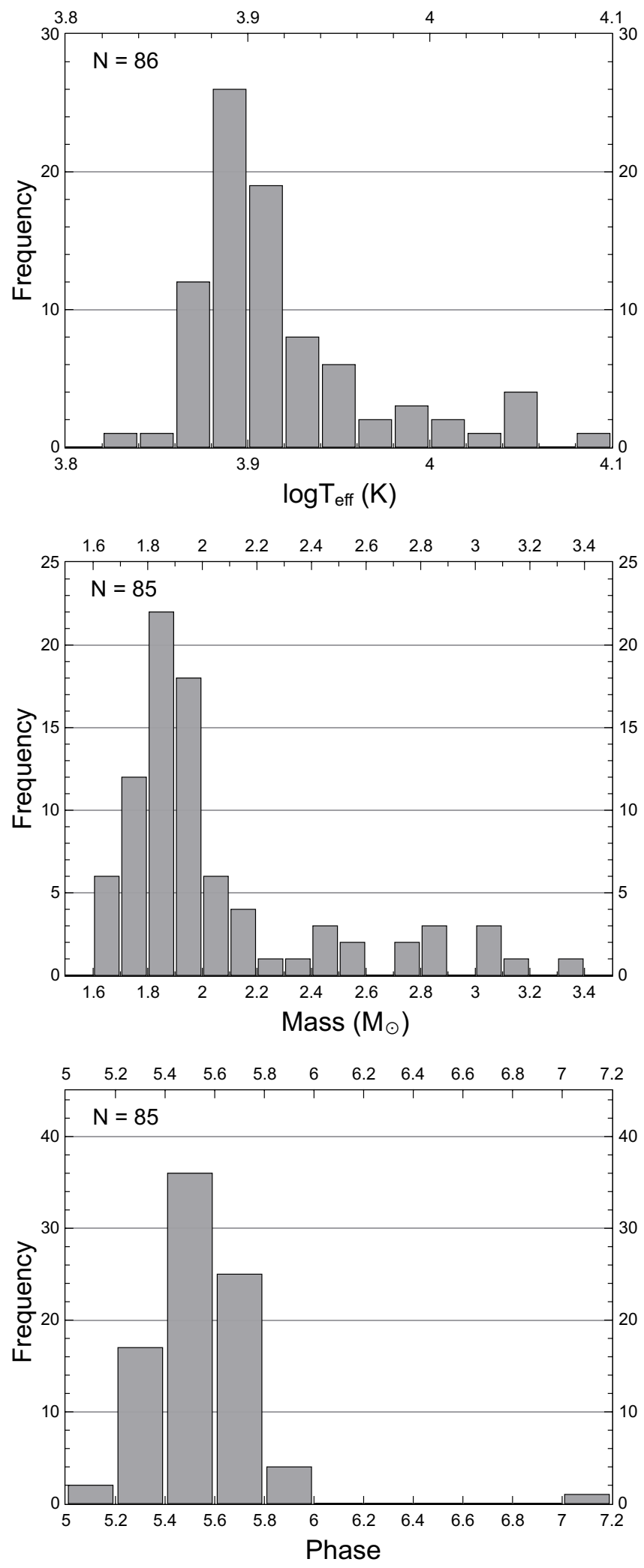

Fig. 4. Distribution of determined effective temperatures (upper panel), estimated masses (middle panel), and estimated evolutionary phases (lower panel). The labeling in the lower panel follows the format of the employed models (Bressan et al. 2012), in which phase 5 represents the zero age main sequence (ZAMS) and phase 6 the terminal age main sequence (TAMS). The large uncertainty in luminosity for one star (ZTFJ232952.58+501457.3) prevented us from calibrating the corresponding mass and phase values. 


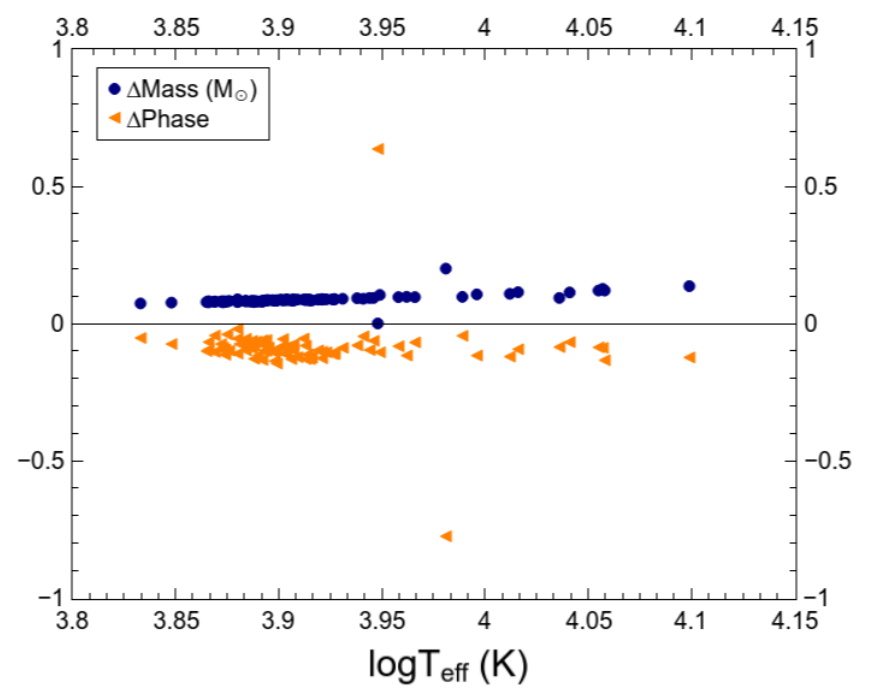

Fig. 5. Comparison of estimated masses and evolutionary phases for $[Z]=0.020$ and $[Z]=0.014$.

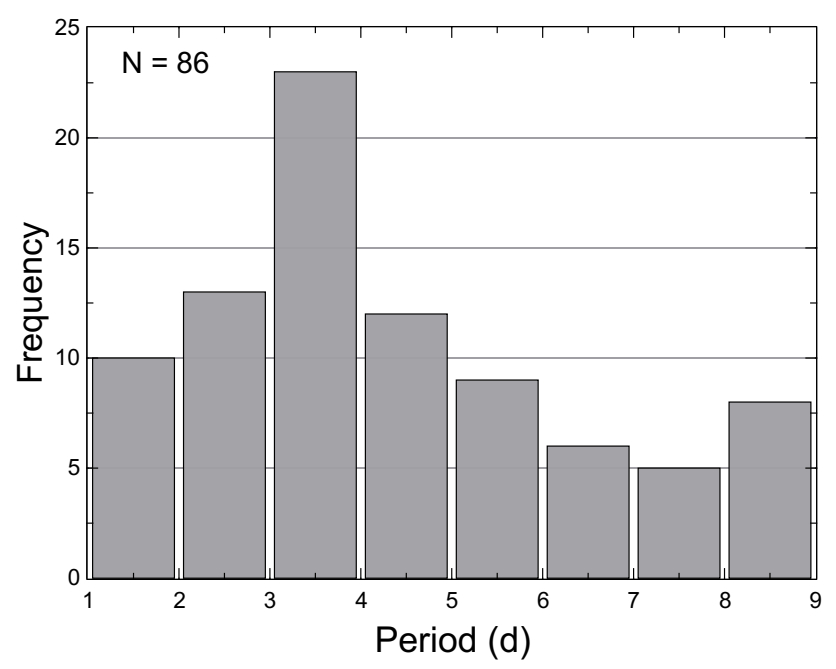

Fig. 6. Distribution of periods among our target star sample, which reflects the selection process described in Sect. 2.

evidence that the catalog classification as RS CVn stars is clearly incorrect (Chen et al. 2020).

A recent article by Asplund et al. (2021) estimated the solar metallicity to $[Z]=0.014$, which is significantly lower than the value used in this work. We have therefore recalculated the corresponding values using $[Z]=0.014$. A comparison of the results based on the two different metallicities is provided in Fig. 5. The mass differences between $[Z]=0.020$ and $[Z]=0.014$ amount on average to about $0.1 M_{\odot}$, in the sense that a lower metallicity results in lower masses. In addition, adopting the lower metallicity value results in a $10 \%$ increase in the derived ages. From a statistical point of view, these differences are negligible.

\subsection{Stars in open cluster fields}

Because the ZTF survey also targets the Galactic disk, we have searched for possible open cluster members among our sample stars following the approach presented in Prišegen et al. (2021). To this end, the angular distance from a given star to the cluster center together with proper motion and parallax con- straints were applied. For the cluster parameters, the catalogs of Cantat-Gaudin et al. (2020), and Dias et al. (2021) were consulted. The astrometric data for our target star sample were taken from the Gaia EDR3 (Gaia Collaboration 2021).

We only found six matches with the newly discovered open clusters of Liu \& Pang (2019, "sc" numbering scheme) and SAI 43 (Glushkova et al. 2010): ZTFJ014156.09+604451.3 (SC 1332), ZTFJ050755.19+495727.2 (SAI 43), ZTFJ060420.41+ 265942.2 (SC 1374), ZTFJ062801.26+162502.0 (SC 1381), ZTFJ 064847.25+ 020447.8 (SC 1392 and SC 1393), and ZTFJ071633.43-020221.4 (SC 1977). In the case of ZTFJ $064847.25+020447.8$ we are unable to decide which of the two clusters it belongs to because the clusters are quite close together and have almost identical parameters. Comparing ages from our estimation (Sect. 3.2) with the corresponding cluster ages, we find excellent agreement. However, we note that the age estimates for the clusters from Liu \& Pang (2019) exhibit quite large errors.

\subsection{Light curves}

The histogram of the periods (Fig. 6) reflects the selection process described in Sect. 2. Although ACV variables can have periods of decades and even longer (Mathys 2019), the detection of periods longer than ten days is hindered by gaps in the data and the rather low-variability amplitude (Hümmerich et al. 2016).

A note is due on the variability of the non-magnetic CP1 and CP3 stars. The CP1 stars are located in the classical pulsation strip and are known to exhibit $\gamma$ Doradus and $\delta$ Scuti variability (Smalley et al. 2017). These variables were already excluded in the selection process (Sect. 2). Both groups also show indications of rotational variability, but with amplitudes of only a few mmags (Balona et al. 2015; Paunzen et al. 2021; Kochukhov et al. 2021). The amplitudes of our variable star sample are much larger (Fig. A.1), which excludes a possible contamination with $\mathrm{CP} 1$ and $\mathrm{CP} 3$ stars, respectively.

In general, concerning the characteristics of the light curves, our target star sample can be divided into four subgroups: (i) $g$ and $r$ data in anti-phase; (ii) the amplitude in $r$ larger than in $g$ and the light curves in phase; (iii) inconsistent light curves in $g$ and $r$ (i.e., they show different shapes); (iv) eclipses with possible additional ACV variability.

In Fig. 7 we present one typical example of each of these subgroups. The full set of light curves is shown in Fig. A.1.

Molnar $(1973,1975)$ reported anti-phase variations between the far-ultraviolet and optical spectral regions suggesting that the global flux redistribution caused by the phase-dependent absorption is the probable cause of the observed phenomena. If so, there is a point or a region in the spectrum where the flux remains almost unchanged (called the null wavelength region) and varies approximately in anti-phase on both sides of it (Mikulášek et al. 2007). The first subgroup of our sample stars (anti-phase variations in $g$ and $r$ ), however, is unusual in that it shows anti-phase variations in the optical region, where in general the light changes are in phase in different photometric passbands (Paunzen et al. 2011). The null wavelength region of our ACV variables has to be somewhere between 4750 and $6200 \AA$, the central wavelengths of the $g$ and $r$ filters. One might speculate that the observed phenomenon is connected to the $5200 \AA$ flux depression. ACV variables with anti-phase variations in the optical region are extremely rare (Gröbel et al. 2017); therefore, these stars are of special interest. Furthermore, it is obvious that this kind of anti-phase variation is a unique characteristic of ACV stars and very much suited to the 
ZTFJ193403.66+632537.7

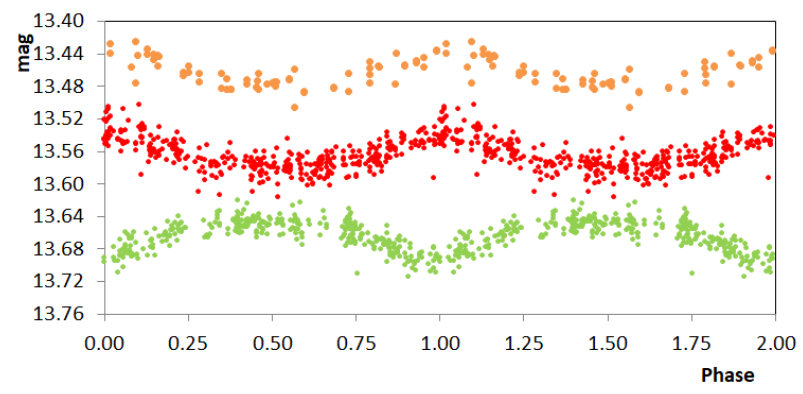

ZTFJ220333.87+481129.9

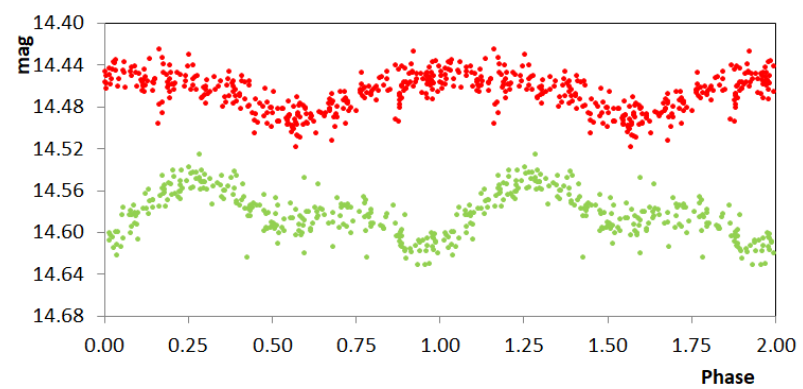

ZTFJ050755.19+495727.2

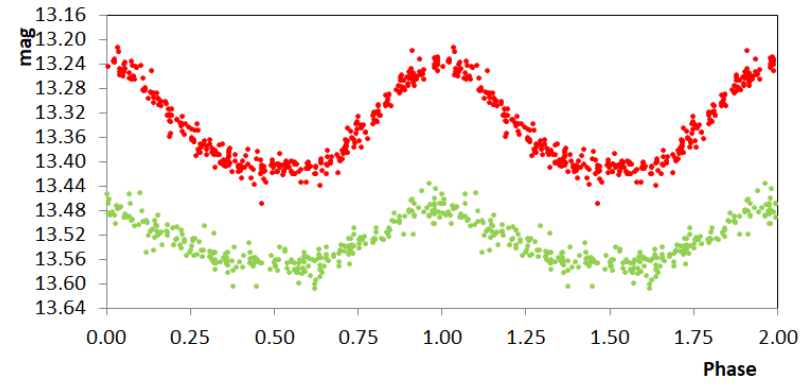

ZTFJ185507.32+323847.6

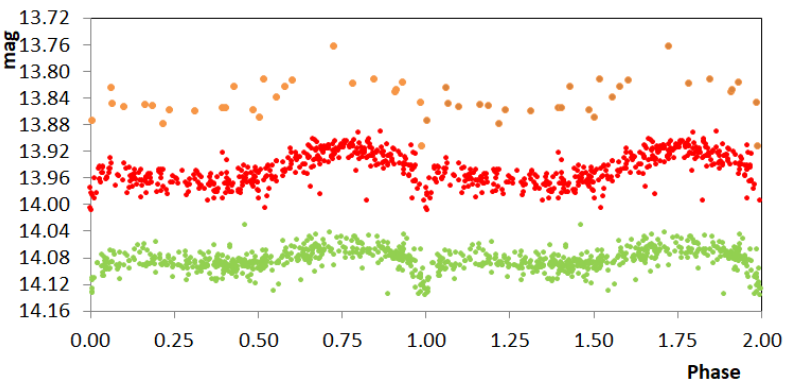

Fig. 7. Example light curves illustrating the four different subgroups among our sample stars: (i) $g$ and $r$ data are in anti-phase (upper left panel), (ii) the amplitude in $r$ is larger than in $g$ (upper right panel), (iii) the light curves in $g$ and $r$ are not consistent (lower left panel), and (iv) eclipses with possible additional ACV variability (lower right panel). Orange, red, and green symbols correspond to $i, r$, and $g$ band data, respectively.

identification of this particular group of stars in massive photometric time series databases that contain observations in different passbands.

Due to a general lack of observations in the red optical region and the near-infrared (Catalano et al. 1998; Janík et al. 2011), the characteristics of the second subgroup (amplitude in $r$ is larger than in $g$ ) are difficult to investigate. The ZTF survey will help analyze this phenomena in more detail, which may turn out to be helpful in the detection of ACV variables because pulsating variables, for example, are expected to show larger amplitudes at shorter wavelengths. However, we note that the light curves are in phase, which is the most common case for ACV variables (Paunzen et al. 2011).

The different characteristics of light curves in different filters and wavelength regions (third subgroup) have been well documented for ACV variables (Manfroid \& Mathys 1986). To date, this phenomenon has almost exclusively been investigated in the narrowband Strömgren photometric system, which covers the spectrum up to $5700 \AA$. The ZTF data allows us to investigate the light curve behavior of ACV variables at even longer wavelengths, which, as the different light curve shapes in the $r$ band demonstrate, opens up an interesting new window on this phenomenon. Additional data in the $i$ band, in particular, will be important to further investigate the light curve characteristics of this subgroup and the correlation with spectral features.

The published overall binary fraction among CP2/4 stars is ranging from 20 to $51 \%$ (Paunzen 2020). However, eclipsing binary systems with such components are extremely rare (Kochukhov et al. 2018; Skarka et al. 2019). We therefore call particular attention to the fourth subgroup of stars (eclipses with possible additional ACV variability). We caution, however, that detailed high-resolution spectroscopy is needed to shed more light on the nature of these objects.
In summary, the quality of the ZTF data is comparable to that of other photometric ground-based surveys like ASAS3, KELT, MASCARA, or SuperWASP (Bernhard et al. 2015a, 2020), while extending to much fainter magnitudes. The ZTF is therefore well suited to searching for new ACV variables and an analysis of their light curve characteristics.

\section{Conclusions}

The ZTF survey is regularly monitoring a large part of the sky in three passbands $(g, r$, and $i)$ to the limiting magnitude of $20.5 \mathrm{mag}$. Observations also include the Galactic disk, which is important to the study of upper main-sequence stars. Extending to much fainter magnitudes, the ZTF is an excellent supplement to the TESS satellite mission.

In this paper we presented a first case study of the potential of the ZTF survey to find and investigate new ACV variables, which are upper main-sequence stars that show variability due to chemical surface spots. Employing selection criteria on the basis of known astrophysical and light curve properties of this group of stars, we identified 86 candidate ACV variables. Fifteen of these candidates could be confirmed as mCP stars with spectra from LAMOST DR7, which proves the viability of our approach.

Zwicky Transient Facility data come in three different passbands, which will allow us to apply the Bayesian photometric imaging technique (Lüftinger et al. 2010; Paunzen et al. 2015b) to a significant number of ACV variables in the future. This method allows the mapping of surface characteristics, and hence the study of the chemical spots. It is similar to a spectroscopic Doppler-imaging analysis, but it is not a tomographic method. The investigation of extensive sets of ground-based spectropolarimetric data for Doppler and magnetic Doppler imaging and photometric data has yielded an excellent correlation of the 
surface maps from photometric and spectroscopic data in terms of spot location and size. The more data are available in different filters, the more accurate the description of the stellar surface can be conducted (Weiss et al. 2020).

Our search for ACV stars in the list of published variables from the ZTF survey has shown that ZTF data are very much suited to the study of this type of variability. However, the available automatic search and classification algorithms employed with the ZTF data do not include the relatively rare class of $\mathrm{ACV}$ variables. To reduce the contamination of other variability classes with ACV variables and to expand the sample size of these stars, it is imperative to be able to correctly identify these stars in survey data of different characteristics. By expanding the current sample of new ACV variables and candidates, we are intending to set up and calibrate a search algorithm to identify these stars, which can then also be applied to any other set of photometric times series. Anti-phase variations, in particular, seem to be an interesting characteristic of a subset of ACV variables that is readily usable for the identification of ACV stars in massive photometric time series databases with observations in different passbands.

Acknowledgements. The authors thank Sebastián Otero (VSX team) for helpful comments. EP acknowledges support by the Erasmus+ programme of the European Union under grant number 2020-1-CZ01-KA203-078200. Based on observations obtained with the Samuel Oschin 48-inch Telescope at the Palomar Observatory as part of the Zwicky Transient Facility project, which is supported by the National Science Foundation under Grant No. AST-1440341 and a collaboration including Caltech, IPAC, the Weizmann Institute for Science, the Oskar Klein Center at Stockholm University, the University of Maryland, the University of Washington, Deutsches Elektronen-Synchrotron and Humboldt University, Los Alamos National Laboratories, the TANGO Consortium of Taiwan, the University of Wisconsin at Milwaukee, and Lawrence Berkeley National Laboratories. Operations are conducted by COO, IPAC, and UW Guoshoujing Telescope (the Large Sky Area Multi-Object Fiber Spectroscopic Telescope LAMOST) is a National Major Scientific Project built by the Chinese Academy of Sciences. Funding for the project has been provided by the National Development and Reform Commission. LAMOST is operated an managed by the National Astronomical Observatories, Chinese Academy of Sciences. This work has made use of data from the European Space Agency (ESA) mission Gaia (https://www.cosmos.esa.int/gaia), processed by the Gaia Data Processing and Analysis Consortium (DPAC, https://www. cosmos.esa.int/web/gaia/dpac/consortium). Funding for the DPAC has been provided by national institutions, in particular the institutions participating in the Gaia Multilateral Agreement. This publication makes use of data products from the Two Micron All Sky Survey, which is a joint project of the University of Massachusetts and the Infrared Processing and Analysis Center/California Institute of Technology, funded by the National Aeronautics and Space Administration and the National Science Foundation.

\section{References}

Andrae, R., Fouesneau, M., Creevey, O., et al. 2018, A\&A, 616, A8

Andrillat, Y., \& Jaschek, C. 1998, A\&A, 337, 512

Arenou, F., Luri, X., Babusiaux, C., et al. 2018, A\&A, 616, A17

Asplund, M., Amarsi, A. M., \& Grevesse, N. 2021, A\&A, 653, A141

Babcock, H. W. 1949, The Observatory, 69, 191

Bailer-Jones, C. A. L., Rybizki, J., Fouesneau, M., Demleitner, M., \& Andrae, R. 2021, AJ, 161, 147

Balona, L. A., Catanzaro, G., Abedigamba, O. P., Ripepi, V., \& Smalley, B. 2015, MNRAS, 448, 1378

Bass, G., \& Borne, K. 2016, MNRAS, 459, 3721

Bayo, A., Rodrigo, C., Barrado Y Navascués, D., et al. 2008, A\&A, 492, 277 Bellm, E. C., Kulkarni, S. R., Graham, M. J., et al. 2019, PASP, 131, 018002 Bernhard, K., Hümmerich, S., \& Paunzen, E. 2015a, Astron. Nachr., 336, 981 Bernhard, K., Hümmerich, S., Otero, S., \& Paunzen, E. 2015b, A\&A, 581, A138 Bernhard, K., Hümmerich, S., \& Paunzen, E. 2020, MNRAS, 493, 3293

Blomme, J., Debosscher, J., De Ridder, J., et al. 2010, ApJ, 713, L204

Bressan, A., Marigo, P., Girardi, L., et al. 2012, MNRAS, 427, 127

Buldgen, G. 2019, Bull. Soc. R. Sci. Liege, 88, 50

Bychkov, V. D., Bychkova, L. V., \& Madej, J. 2003, A\&A, 407, 631

Cantat-Gaudin, T., Anders, F., Castro-Ginard, A., et al. 2020, A\&A, 640, A1
Castelli, F., \& Kurucz, R. L. 2003, in Modelling of Stellar Atmospheres, eds. N. Piskunov, W. W. Weiss, \& D. F. Gray, 210, A20

Castelli, F., Gratton, R. G., \& Kurucz, R. L. 1997, A\&A, 318, 841

Catalano, F. A., Leone, F., \& Kroll, R. 1998, A\&AS, 131, 63

Chen, Y., Girardi, L., Bressan, A., et al. 2014, MNRAS, 444, 2525

Chen, X., Wang, S., Deng, L., et al. 2020, ApJS, 249, 18

Cui, X.-Q., Zhao, Y.-H., Chu, Y.-Q., et al. 2012, Res. Astron. Astrophys., 12, 1197

Cunha, M. S., Antoci, V., Holdsworth, D. L., et al. 2019, MNRAS, 487, 3523

Dias, W. S., Monteiro, H., Moitinho, A., et al. 2021, MNRAS, 504, 356

Eaton, J. A., Henry, G. W., Bell, C., \& Okorogu, A. 1993, AJ, 106, 1181

Flower, P. J. 1996, ApJ, 469, 355

Gaia Collaboration (Brown, A. G. A., et al.) 2021, A\&A, 649, A1

Garrison, R. F., \& Gray, R. O. 1994, AJ, 107, 1556

Glushkova, E. V., Koposov, S. E., Zolotukhin, I. Y., et al. 2010, Astron. Lett., 36, 75

Gray, R. O., \& Corbally, C., Jr. 2009, Stellar Spectral Classification (Princeton: Princeton University Press)

Gray, R. O., \& Garrison, R. F. 1987, ApJS, 65, 581

Gray, R. O., \& Garrison, R. F. 1989a, ApJS, 69, 301

Gray, R. O., \& Garrison, R. F. 1989b, ApJS, 70, 623

Green, G. M., Schlafly, E., Zucker, C., Speagle, J. S., \& Finkbeiner, D. 2019, ApJ, 887, 93

Gröbel, R., Hümmerich, S., Paunzen, E., \& Bernhard, K. 2017, New Astron., 50, 104

Guthnik, P., \& Prager, R. 1914, Veröffentlichungen der Königlichen Sternwarte zu Berlin-Babelsberg (Berlin: Dümmler)

Henden, A., \& Munari, U. 2014, Contrib. Astron. Obs. Skaln. Pleso, 43, 518

Hubrig, S., Järvinen, S. P., Madej, J., et al. 2018, MNRAS, 477, 3791

Hümmerich, S., Paunzen, E., \& Bernhard, K. 2016, AJ, 152, 104

Hümmerich, S., Bernhard, K., \& Paunzen, E. 2018, Contrib. Astron. Obs. Skaln. Pleso, 48, 277

Hümmerich, S., Paunzen, E., \& Bernhard, K. 2020, A\&A, 640, A40

Jagelka, M., Mikulášek, Z., Hümmerich, S., \& Paunzen, E. 2019, A\&A, 622, A199

Janík, J., Mikulášek, Z., Szász, G., et al. 2011, Magnetic Stars, 476

Kochukhov, O., Johnston, C., Alecian, E., Wade, G. A., \& BinaMIcS Collaboration 2018, MNRAS, 478, 1749

Kochukhov, O., Khalack, V., Kobzar, O., et al. 2021, MNRAS, 506, 5328

Kozhevnikova, A. V., \& Alekseev, I. Y. 2015, Astron. Rep., 59, 937

Krtička, J. 2016, A\&A, 594, A75

Krtička, J., Mikulášek, Z., Zverko, J., \& Žižńovský, J. 2007, in Physics of Magnetic Stars, eds. I. I. Romanyuk, D. O. Kudryavtsev, O. M. Neizvestnaya, \& V. M. Shapoval, 310

Krtička, J., Mikulášek, Z., Henry, G. W., et al. 2009, A\&A, 499, 567

Kügler, S. D., Gianniotis, N., \& Polsterer, K. L. 2015, MNRAS, 451, 3385

Kupka, F., Paunzen, E., \& Maitzen, H. M. 2003, MNRAS, 341, 849

Kupka, F., Paunzen, E., Iliev, I. K., \& Maitzen, H. M. 2004, MNRAS, 352, 863

Lenz, P., \& Breger, M. 2005, Commun. Asteroseismol., 146, 53

Liu, L., \& Pang, X. 2019, ApJS, 245, 32

Lüftinger, T., Fröhlich, H. E., Weiss, W. W., et al. 2010, A\&A, 509, A43

Malkov, O. Y., Sichevskij, S. G., \& Kovaleva, D. A. 2010, MNRAS, 401, 695

Manfroid, J., \& Mathys, G. 1986, A\&AS, 64, 9

Masci, F. J., Laher, R. R., Rusholme, B., et al. 2019, PASP, 131, 018003

Mathys, G. 2019, in Southern Horizons in Time-Domain Astronomy, ed. R. E. Griffin, 339, 106

Michaud, G., Megessier, C., \& Charland, Y. 1981, A\&A, 103, 244

Mikulášek, M., Zverko, J., Krtička, J., et al. 2007, in Physics of Magnetic Stars, eds. I. I. Romanyuk, D. O. Kudryavtsev, O. M. Neizvestnaya, \& V. M Shapoval, 300

Molnar, M. R. 1973, ApJ, 179, 527

Molnar, M. R. 1975, AJ, 80, 137

Netopil, M., Paunzen, E., Maitzen, H. M., North, P., \& Hubrig, S. 2008, A\&A, 491, 545

Netopil, M., Paunzen, E., Hümmerich, S., \& Bernhard, K. 2017, MNRAS, 468, 2745

Ofek, E. O., Soumagnac, M., Nir, G., et al. 2020, MNRAS, 499, 5782

Paunzen, E. 2015, A\&A, 580, A23

Paunzen, E. 2020, Contrib. Astron. Obs. Skaln. Pleso, 50, 570

Paunzen, E., \& Vanmunster, T. 2016, Astron. Nachr., 337, 239

Paunzen, E., Stütz, C., \& Maitzen, H. M. 2005, A\&A, 441, 631

Paunzen, E., Schnell, A., \& Maitzen, H. M. 2006, A\&A, 458, 293

Paunzen, E., Netopil, M., Rode-Paunzen, M., Handler, G., \& Božić, H. 2015a, A\&A, 575, A24

Paunzen, E., Fröhlich, H. E., Netopil, M., Weiss, W. W., \& Lüftinger, T. 2015b, A\&A, 574, A57

Paunzen, E., Hensberge, H., Maitzen, H. M., et al. 2011, A\&A, 525, A16

Paunzen, E., Netopil, M., \& Herdin, A. 2017, Bulg. Astron. J., 26, 45 
N. Faltová et al.: A case study of ACV variables discovered in the Zwicky Transient Facility survey

Paunzen, E., Handler, G., Hoňková, K., et al. 2018, Res. Astron. Astrophys., 18, 135

Paunzen, E., Hümmerich, S., \& Bernhard, K. 2021, A\&A, 645, A34

Preston, G. W. 1974, ARA\&A, 12, 257

Prišegen, M., Piecka, M., Faltová, N., Kajan, M., \& Paunzen, E. 2021, A\&A, 645, A13

Qin, L., Luo, A. L., Hou, W., et al. 2019, ApJS, 242, 13

Romanyuk, I. I. 2014, in Putting A Stars into Context: Evolution, Environment, and Related Stars, eds. G. Mathys, E. R. Griffin, O. Kochukhov, R. Monier, \& G. M. Wahlgren, 371

Saffe, C., Levato, H., \& López-García, Z. 2005, Rev. Mex. Astron. Astrofis., 41 415
Samus', N. N., Kazarovets, E. V., Durlevich, O. V., Kireeva, N. N., \& Pastukhova, E. N. 2017, Astron. Rep., 61, 80

Sikora, J., David-Uraz, A., Chowdhury, S., et al. 2019, MNRAS, 487, 4695

Skarka, M., Kabáth, P., Paunzen, E., et al. 2019, MNRAS, 487, 4230

Skrutskie, M. F., Cutri, R. M., Stiening, R., et al. 2006, AJ, 131, 1163

Smalley, B., Antoci, V., Holdsworth, D. L., et al. 2017, MNRAS, 465, 2662

Stibbs, D. W. N. 1950, MNRAS, 110, 395

Vagnozzi, S. 2019, Atoms, 7, 41

Weiss, W. W., Fröhlich, H. E., Kallinger, T., et al. 2020, A\&A, 642, A64

Zhao, G., Zhao, Y., Chu, Y., Jing, Y., \& Deng, L. 2012, ArXiv e-prints [arXiv:1206.3569] 
Appendix A: Essential data and light curves for our sample stars

Table A.1 lists essential data for our sample stars. It is organised as follows:

- Column 1: ZTF ID.

- Column 2: Identifier from Gaia EDR3.

- Column 3: Right ascension (J2000). Positional information was taken from GAIA EDR3 (Gaia Collaboration 2021).

- Column 4: Declination (J2000).
- Column 5: Period from Chen et al. (2020).

- Column 6: $G$ magnitude (Gaia DR2).

- Column 7: $G$ magnitude error (Gaia DR2).

- Column 8: Parallax (Gaia EDR3).

- Column 9: Parallax error.

- Column 10: Mean effective temperature.

- Column 11: Mean effective temperature error.

- Column 12: Luminosity.

- Column 13: Luminosity error.

- Column 14: Mass.

- Column 15: Evolutionary phase. 
N. Faltová et al.: A case study of ACV variables discovered in the Zwicky Transient Facility survey

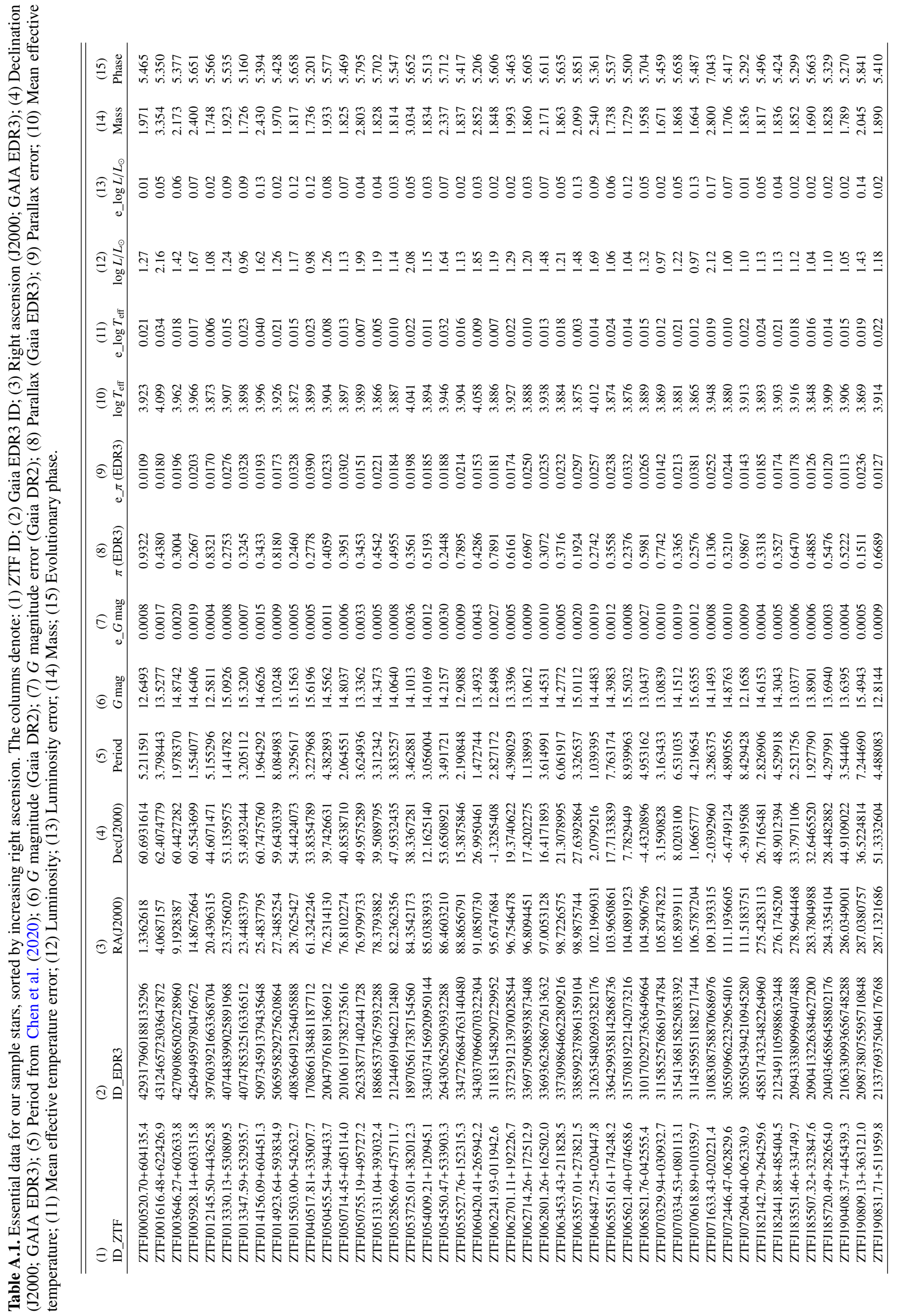




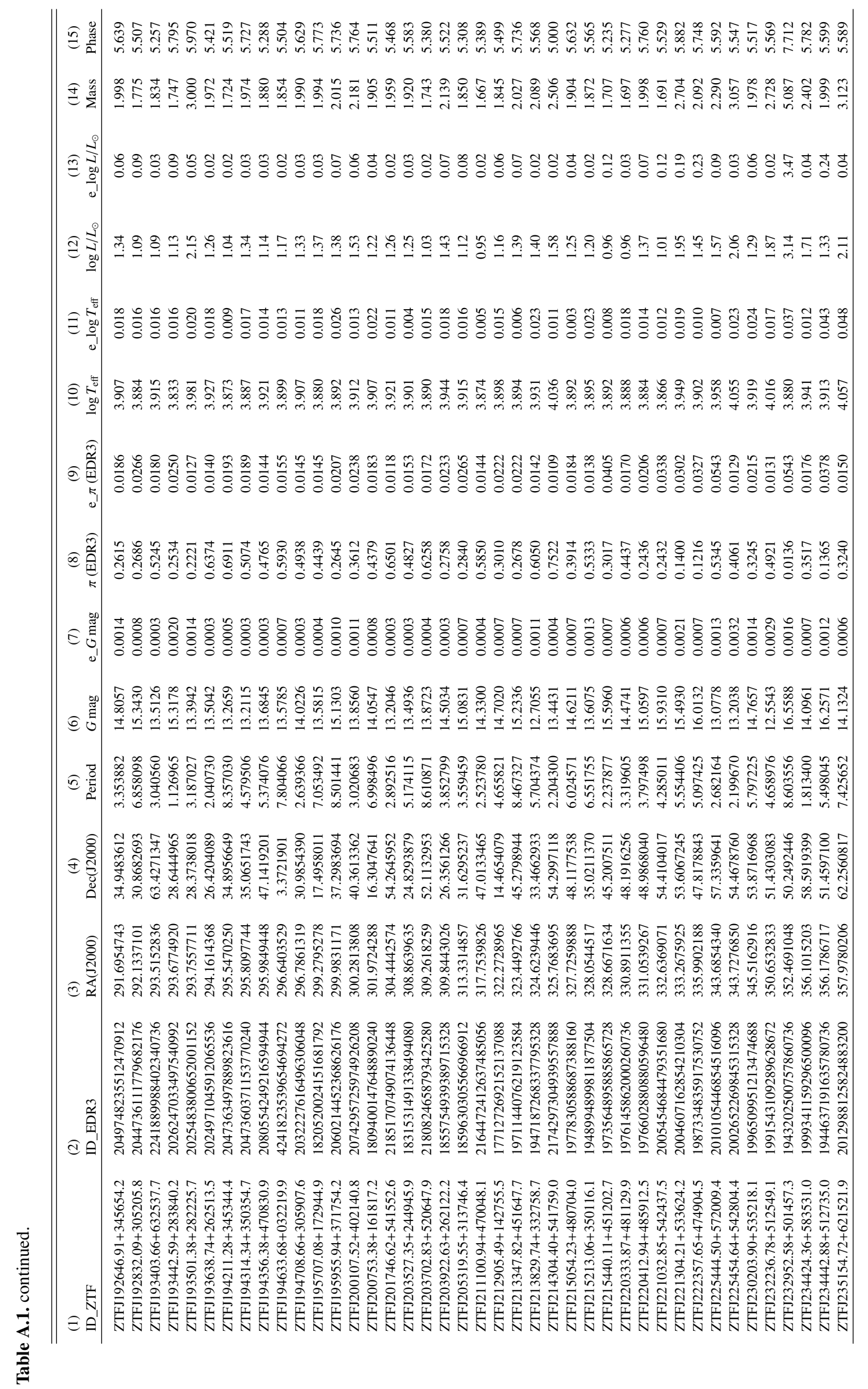


N. Faltová et al.: A case study of ACV variables discovered in the Zwicky Transient Facility survey
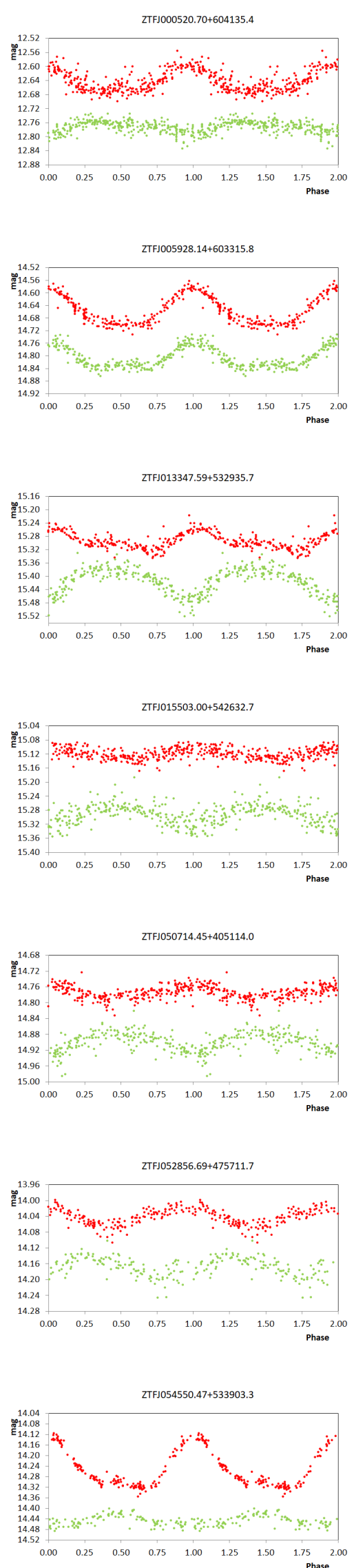
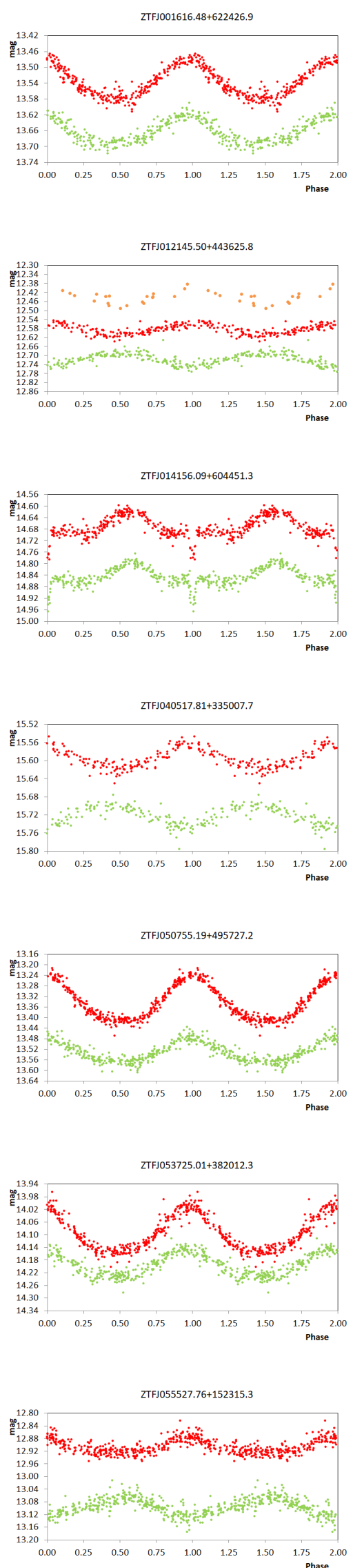
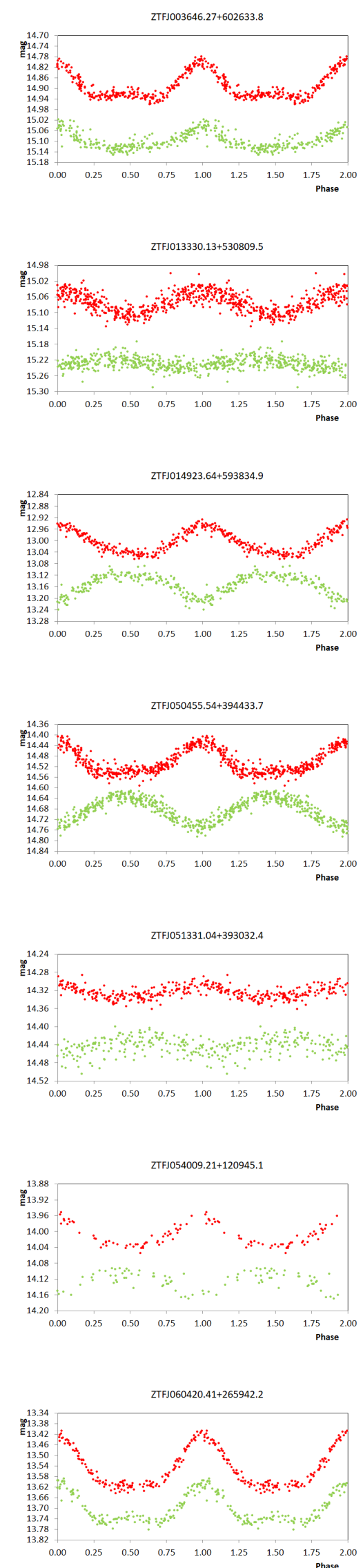

Fig. A.1. Phased light curves of our target stars in all available passbands. Orange, red, and green symbols correspond to $i, r$, and $g$ band data, respectively. 
A\&A 656, A125 (2021)
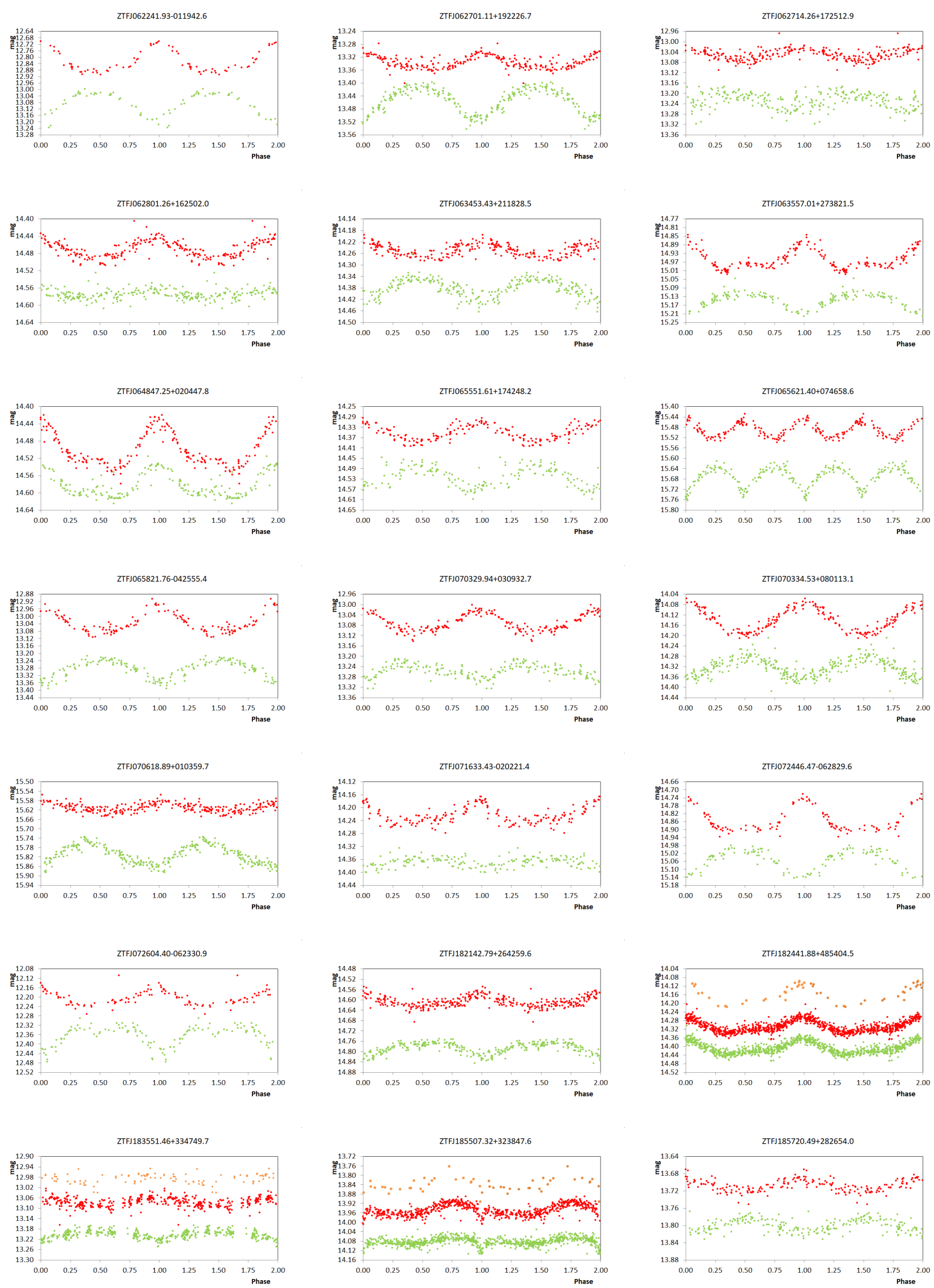

Fig. A.1. continued. 
N. Faltová et al.: A case study of ACV variables discovered in the Zwicky Transient Facility survey
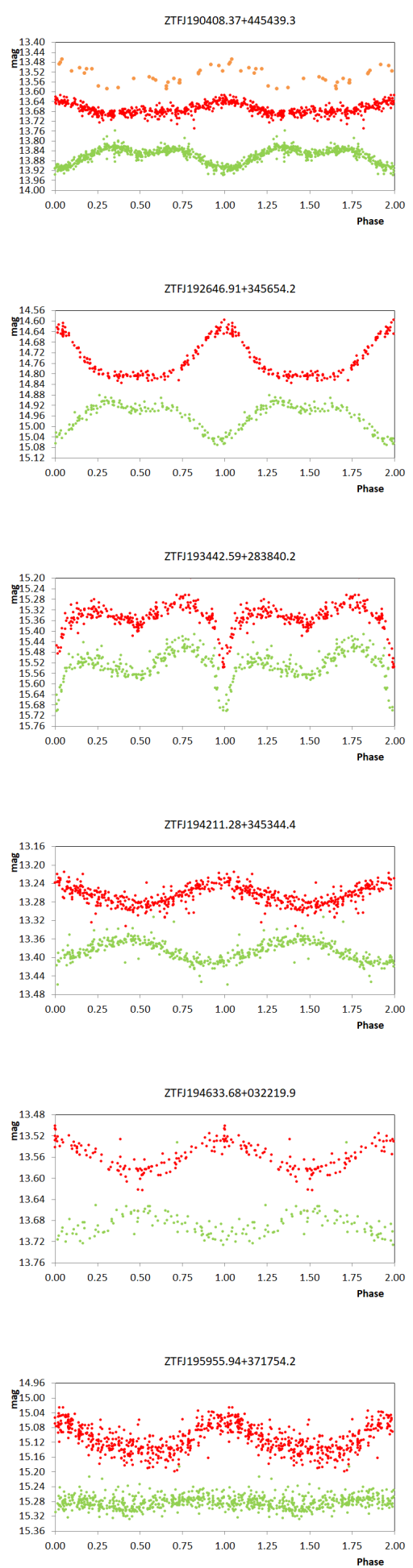

ZTF $201746.62+541552.6$

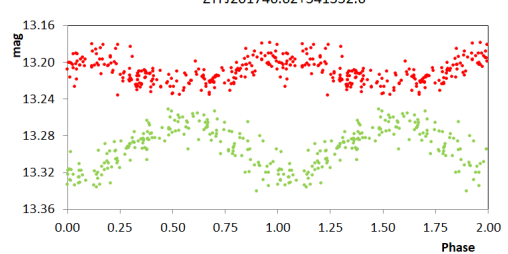

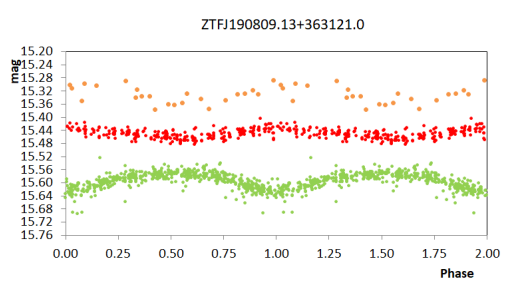

ZTF 192832.09+305205.8
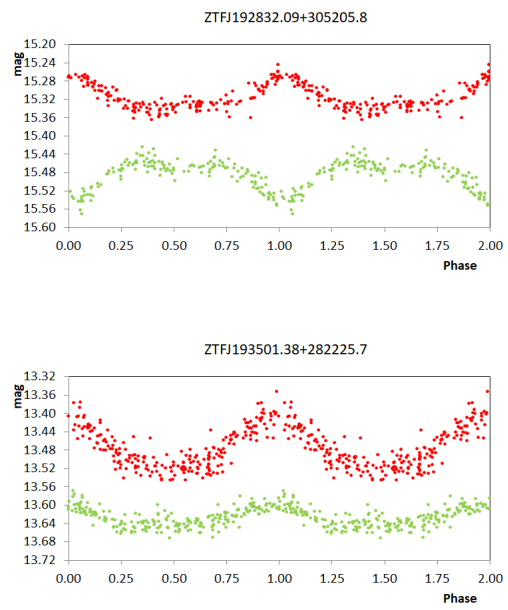

ZTFJ194314.34+350354.7

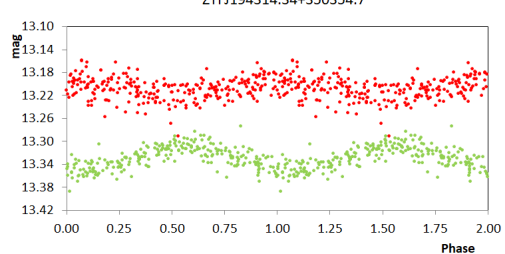

ZTF194708.66+305907.6
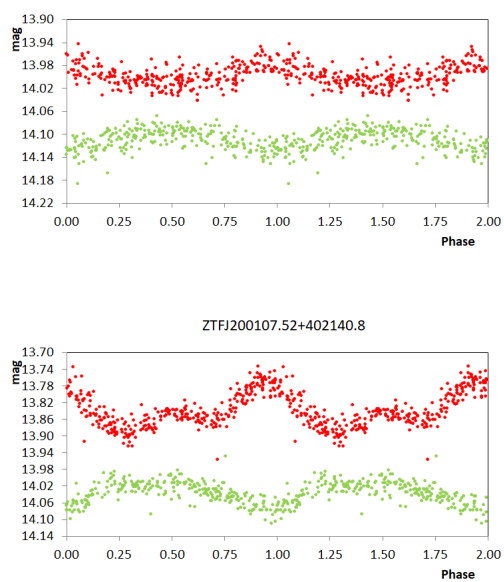

ZTFJ203527.35+244945.9

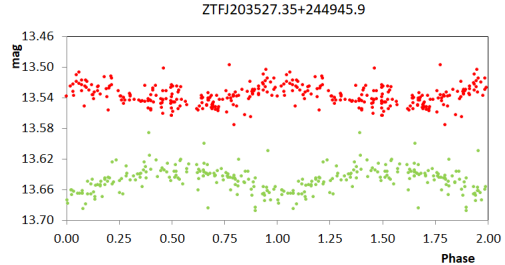

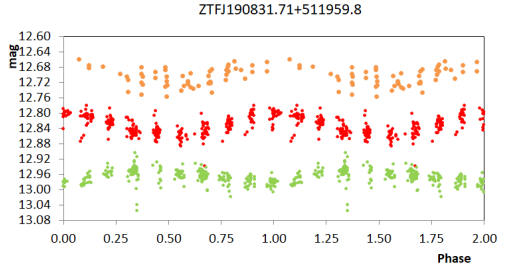

ZTF 193403.66+632537.7
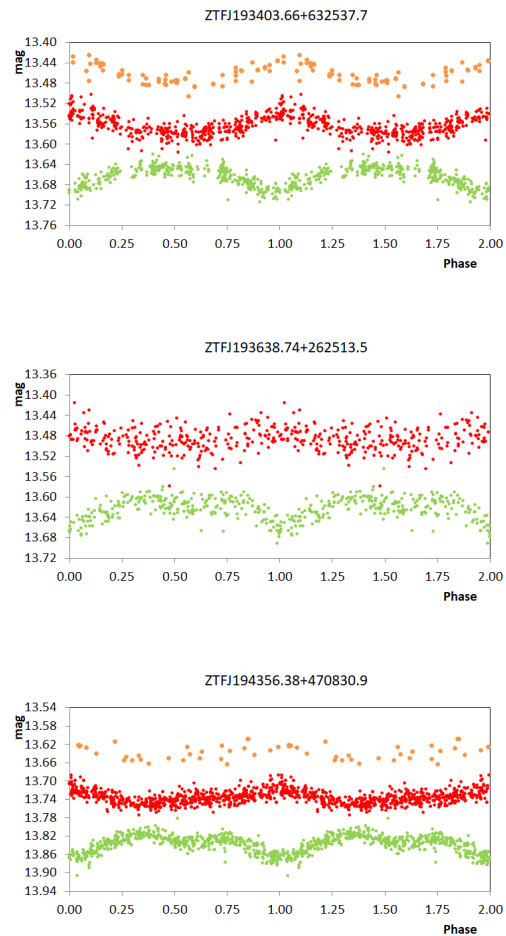

ZTF 195707.08+172944.9
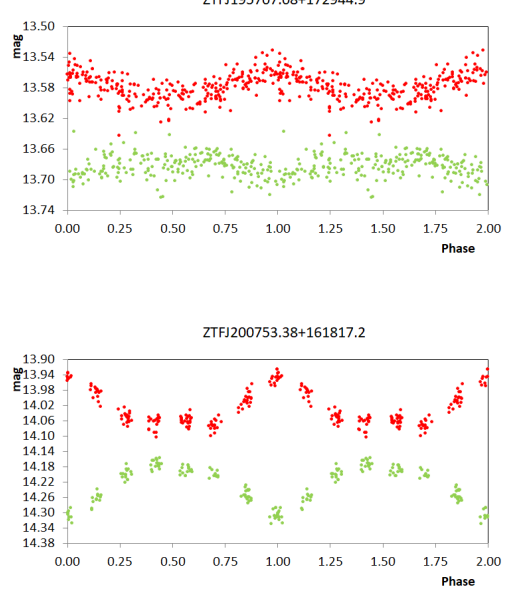

ZTF 203702.83+520647.9

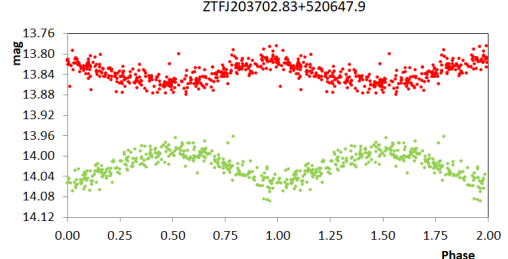

Fig. A.1. continued. 

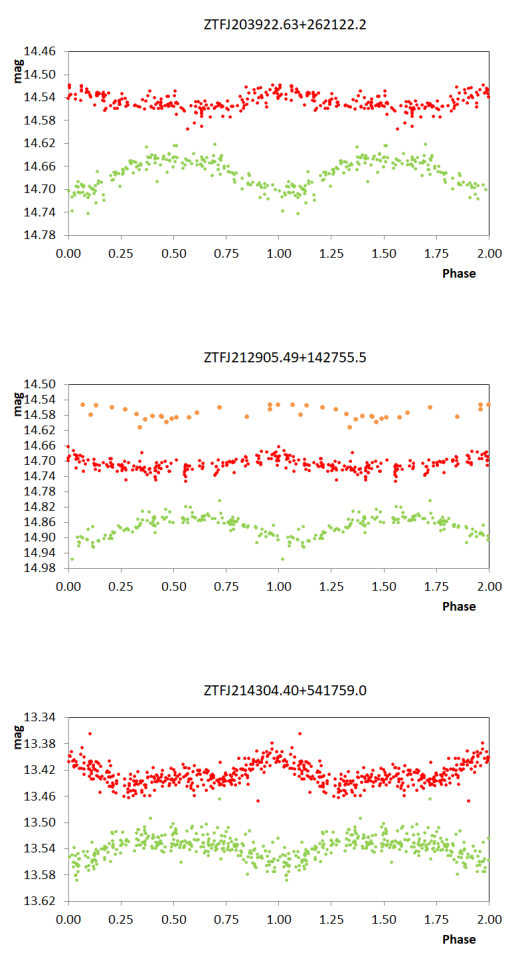

ZTFJ215440.11+451202.7
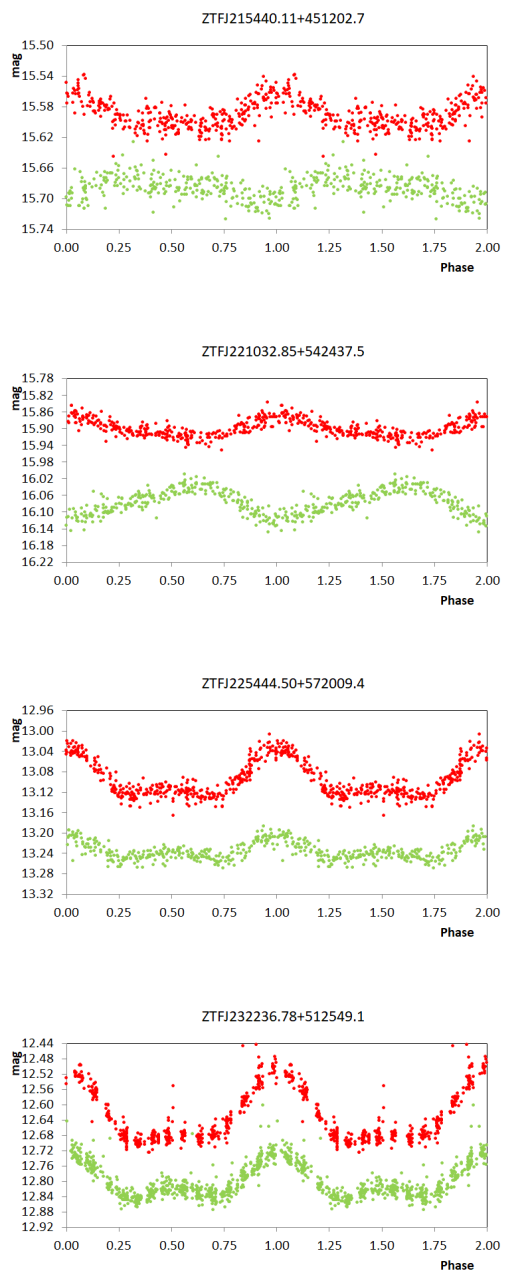

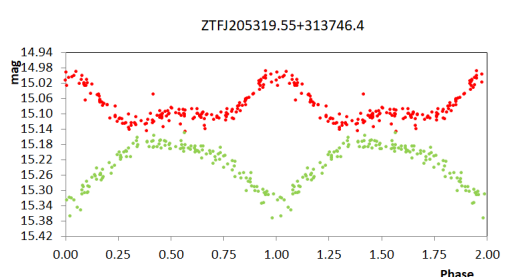

ZTF213347.82+451647.7
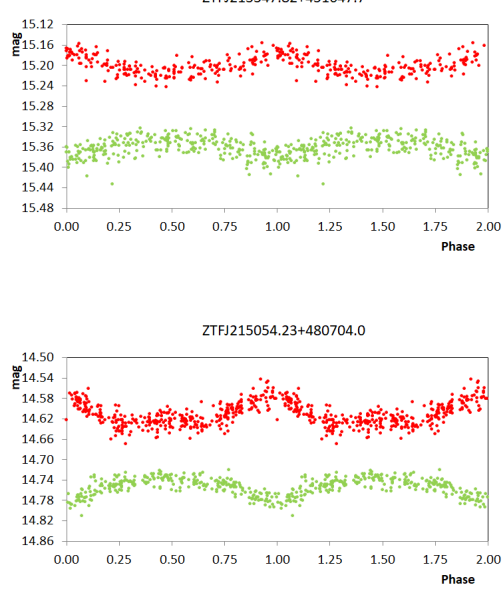

ZTFJ220333.87+481129.9
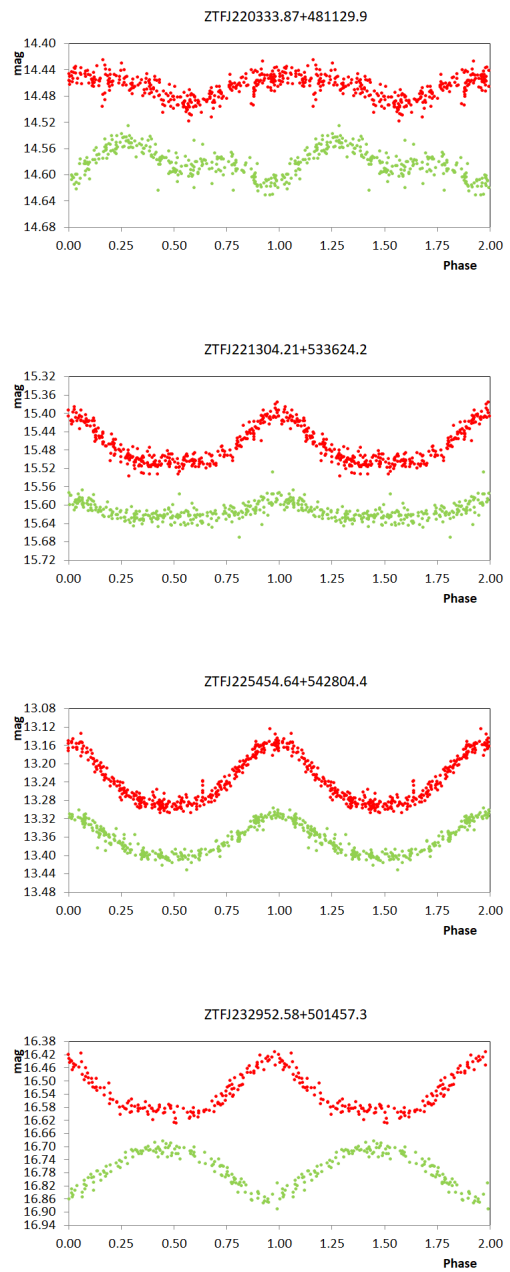

ZTFJ211100.94+470048.1
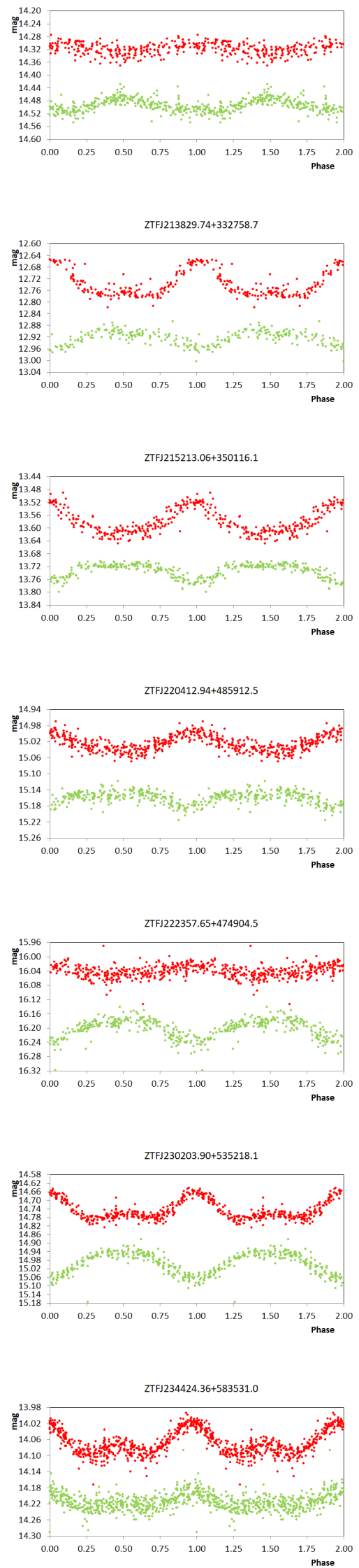

Fig. A.1. continued. 
N. Faltová et al.: A case study of ACV variables discovered in the Zwicky Transient Facility survey
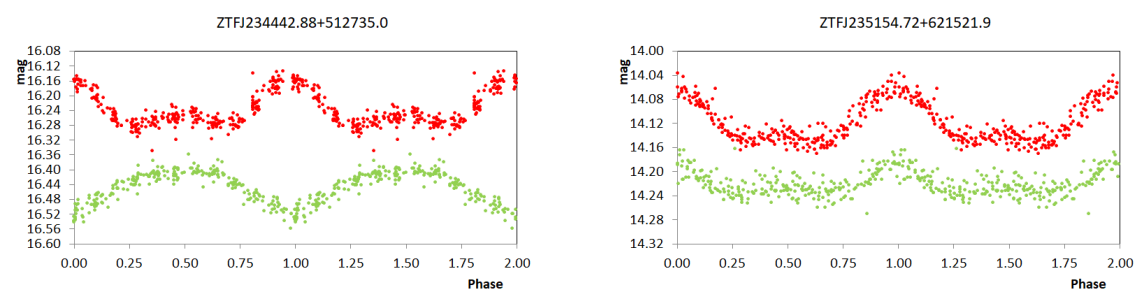

Fig. A.1. continued. 